\title{
Collective Analysis of Qualitative Data
}

Simonsen, Jesper; Friberg, Karin

Published in:

Situated Design Methods

Publication date:

2014

Document Version

Early version, also known as pre-print

Citation for published version (APA):

Simonsen, J., \& Friberg, K. (2014). Collective Analysis of Qualitative Data. In J. Simonsen, C. Svabo, S. M.

Strandvad, K. Samson, M. Hertzum, \& O. E. Hansen (Eds.), Situated Design Methods (pp. 99-117). MIT Press. Design thinking, design theory

\section{General rights}

Copyright and moral rights for the publications made accessible in the public portal are retained by the authors and/or other copyright owners and it is a condition of accessing publications that users recognise and abide by the legal requirements associated with these rights.

- Users may download and print one copy of any publication from the public portal for the purpose of private study or research.

- You may not further distribute the material or use it for any profit-making activity or commercial gain.

- You may freely distribute the URL identifying the publication in the public portal.

Take down policy

If you believe that this document breaches copyright please contact rucforsk@kb.dk providing details, and we will remove access to the work immediately and investigate your claim. 
PREPRINT: Simonsen, J., and K. Friberg (2014): "Collective Analysis

of Qualitative Data," in J. Simonsen, C. Svabo, S. M. Strandvad, K.

Samson, M. Hertzum, and O. E. Hansen (Eds.): Situated Design

Methods, MIT Press, Boston, pp. 99-117.

\title{
Collective Analysis of Qualitative Data
}

Jesper Simonsen and Karin Friberg

\begin{abstract}
What. Many students and practitioners do not know how to systematically process qualitative data once it is gathered -at least, not as a collective effort. This chapter presents two workshop techniques - affinity diagramming and diagnostic mapping - that support collective analysis of large amounts of qualitative data. Affinity diagramming is used to make collective analysis and interpretations of qualitative data to identify core problems that need to be addressed in the design process. Diagnostic mapping supports collective interpretation and description of these problems and how to intervene in them. The techniques are explained through a case where they were used to analyze why a new electronic medical record system introduced life-threatening situations for patients.

Why. Collective analyses offer all participants a voice, visualizes their contributions, combines different actors' perspectives, and supports that the result of the interpretation is anchored to the participating actors. Combining the techniques is a powerful way to analyze and intervene in situations prior or after the introduction of new information technologies.

Where. The techniques are general tools that might be widely applied in different domains. In particular, collective analysis can be used to identify, understand, and act upon complex design problems that emerge, for example, after the introduction of new technologies. Such problems might be hard to clarify and the basis for the analysis is often large amounts of unstructured qualitative data - for example, from numerous interviews.

How. Affinity diagrams visualize 'core categories' from the body of data. Diagnostic mapping visualizes problems, their causes and consequences along with any ideas for solutions. Both techniques are used in workshop form where the participants jointly analyze, discuss, and interpret the empirical material visualized by pads of adhesive notes.
\end{abstract}

\section{Introduction}

Academic work often includes analysis of large amounts of unstructured and qualitative data. Consider the situation where a student group or a research team at a university have conducted a number of interviews: How do the project group make a systematic analysis of the resulting interview material, i.e. audio recordings, transcripts, personal notes and thoughts? In some 
situations this is simply done by having a meeting where the participants from the project group meet and discuss what each of them believe to be important contributions from the interviews. But then the individual participants risk approaching the discussion solely from their own interpretation and perspective of the data, promoting their own ideas and agenda and interests, and perhaps ignoring or misunderstanding others' ideas and input. In this case maybe only by chance will general insights emerge as a result of what the project group together as a collective are able to interpret and conclude.

This chapter offers a systematic method for conducting a collective analysis of qualitative data. The main point is to facilitate a collective process of interpretation (vs. an individual interpretation process). The collective interpretation process includes an initial inductive collective analysis of the data material based on Grounded Theory (Glaser and Strauss 1967), and a subsequent abductive collective process supporting an innovative and designoriented intervention for change. The former is supported by the affinity diagramming technique (Brassard 1989) while the latter is facilitated by the diagnostic mapping technique (Lanzara and Mathiassen 1985).

The combined use of the two techniques has been part of the curriculum at a university design course held by one of the authors for more than a decade. The experience from the course has shown that the students are able to apply the techniques quickly and independently while also conducting high-quality collective analysis. Using the techniques for the first time typically provides the students with a great 'aha'experience.

In the following, the background and the main ideas behind affinity diagramming and diagnostic mapping is outlined. Then a case is described, analyzing a complex problematic situation involving a new electronic medical record (EMR) system. The system was introduced in order to obtain a safer medication procedure. After the implementation, the designers undertook a questionnaire survey to learn about the user experience. To their astonishment, one-third of the respondents reported that the medication procedure had become less safe, sometimes leading to life-threatening situations. This raised an immediate design problem: Why is the new EMR-supported medication process not experienced as being unequivocally safer? The case describes how large amounts of qualitative data were gathered and analyzed by affinity diagramming and diagnostic mapping to investigate why the system introduced life-threatening situations for patients. The chapter then gives a concrete hands-on suggestion for how collective analysis can be applied in student projects. Finally, the chapter closes with a summarizing conclusion.

\section{Background}

The affinity diagramming technique has Japanese origins, was originally described by Brassard (1989), and has been widely used in design methods, 
including Contextual Design (Beyer and Holtzblatt 1998, 154 ff.) and the MUST method (Bødker et al. Chapter 4; Bødker et al. 2004, 253).

Affinity diagramming is theoretically rooted in Grounded Theory. Grounded Theory was developed by Glaser and Strauss (Glaser and Strauss 1967; Glaser 1992) as a strategy for analyzing qualitative data. The theory is 'grounded,' which means that it includes descriptions and explanations made through an analysis of empirical data - i.e. it is grounded in this data: "grounded theory is derived from data and then illustrated by characteristic examples of data" (Glaser and Strauss 1967, 5).

Grounded Theory forms an inductive process where numerous single observations from the data are analyzed in a bottom-up approach to form more general conclusions. The theory analyzes qualitative data from, for example, transcripts or elaborated summaries of interviews: First, this data is coded: i.e. key points of interest in the data are marked, for example by underlining a word or text passage in an interview transcript. Second, through a process of constant comparison of codes, low-level categories (ibid. p. 36) emerge from the data as groups of codes of similar content. Third, low-level categories might evolve into high-level categories or core categories (Glaser 1992, 75). High-level categories can, for example, identify a problematic situation that is described and elaborated through all the associated codes. The goal of Grounded Theory is "the generation of theory around a core category [...that explain the...] patterns of behavior" (ibid., 75) that are relevant or problematic.

Affinity diagramming helps to create an overview over large amounts of data through an inductive process as described by Grounded Theory: "Building an affinity is inductive reasoning at its purest. To put up one note, then for everyone to look for other notes that seem to go with it" (Brassard 1989, 30). The starting point is a number of isolated statements (codes) written on pads of adhesive notes. The adhesive notes are distributed and grouped on a board (see Figure 1). The statements (codes) on the notes are interpreted and assessed to clarify and determine which group (category) an adhesive note might belong to. This principle of "making the data talk" is central to Grounded Theory and the affinity diagramming: The point is to enable categories to emerge as part of the process of analyzing and comparing of the data - rather than analyzing data based on predefined categories or hypotheses (Glaser and Strauss 1967, 36; Glaser 1992, 39). The result is an affinity diagram outlining low-level and high-level categories. Affinity diagramming is a workshop technique offering a resource for situated action (Suchman, 2007). The core aspect of the technique is that it is data-driven, i.e. driven by the empirical data at hand. The technique stipulates how to work with these data while the actual workshop processes as well as the results are totally dependent on the available qualitative data. 


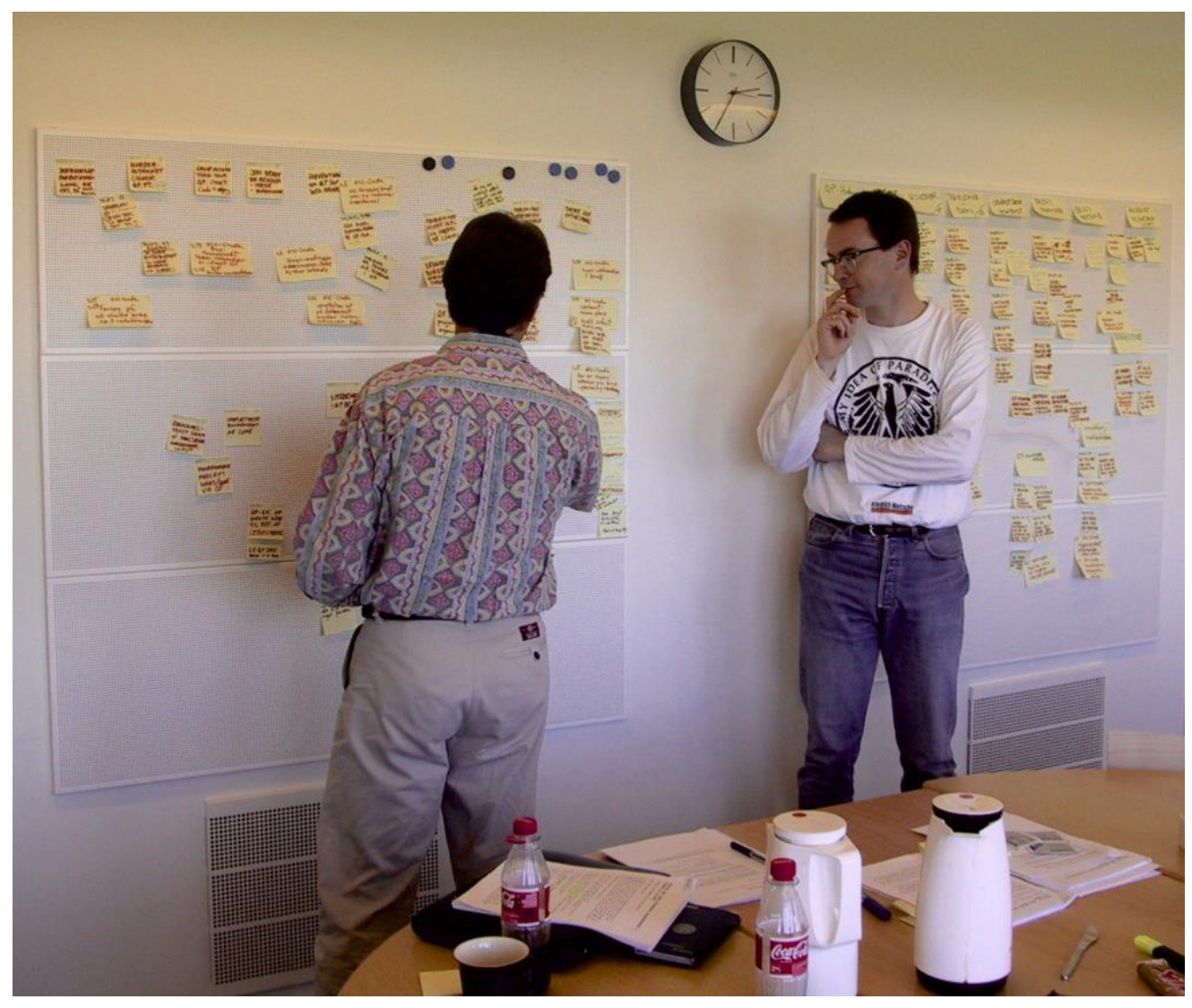

Figure 1: Photo depicting an affinity diagram workshop.

The problem mapping technique can be used to analyze problems that have been identified in the affinity diagram. Problem mapping supports an abductive reasoning: a process of suggesting and stating hypotheses explaining problems as well as suggesting possible ways to solve them. The technique was originally described by Lanzara and Mathiassen (1985), has been applied to information technology design methods (Andersen et al. 1990, 136 ff.; Bødker et al. 2004, 277ff.), used to support the principle of 'anchoring visions' (see Bødker et al., Chapter 4), and used to engage top management in design projects (Simonsen, 2007). Diagnostic mapping is used to analyze (diagnose) problematic situations. When it is combined with affinity diagramming the technique takes a starting point in problems identified in the affinity diagram as core categories. For each of these problems the technique enables a structuring of arguments, explaining their causes, consequences, and ideas for solutions. The argument chains are built using adhesive notes from the affinity diagram (when appropriate) as well as new added statements (hypotheses) not directly evident in the data (see Figure 2). The aim of diagnostic mapping is to generate ideas for how to solve problems. The technique pursues the explanation of problems and how to intervene in them. This way, the technique supplements an inductive Grounded Theory-based analysis with an abductive and design-oriented intervention. Just like affinity diagramming, 
diagnostic mapping constitutes a resource for situated action (Suchman, 2007). The core aspect of the technique is that it is stakeholder-involving. The technique offers an agenda and a visualization tool for the workshop while the processes as well as the results are totally dependent on reflections and discussions raised by the participants.

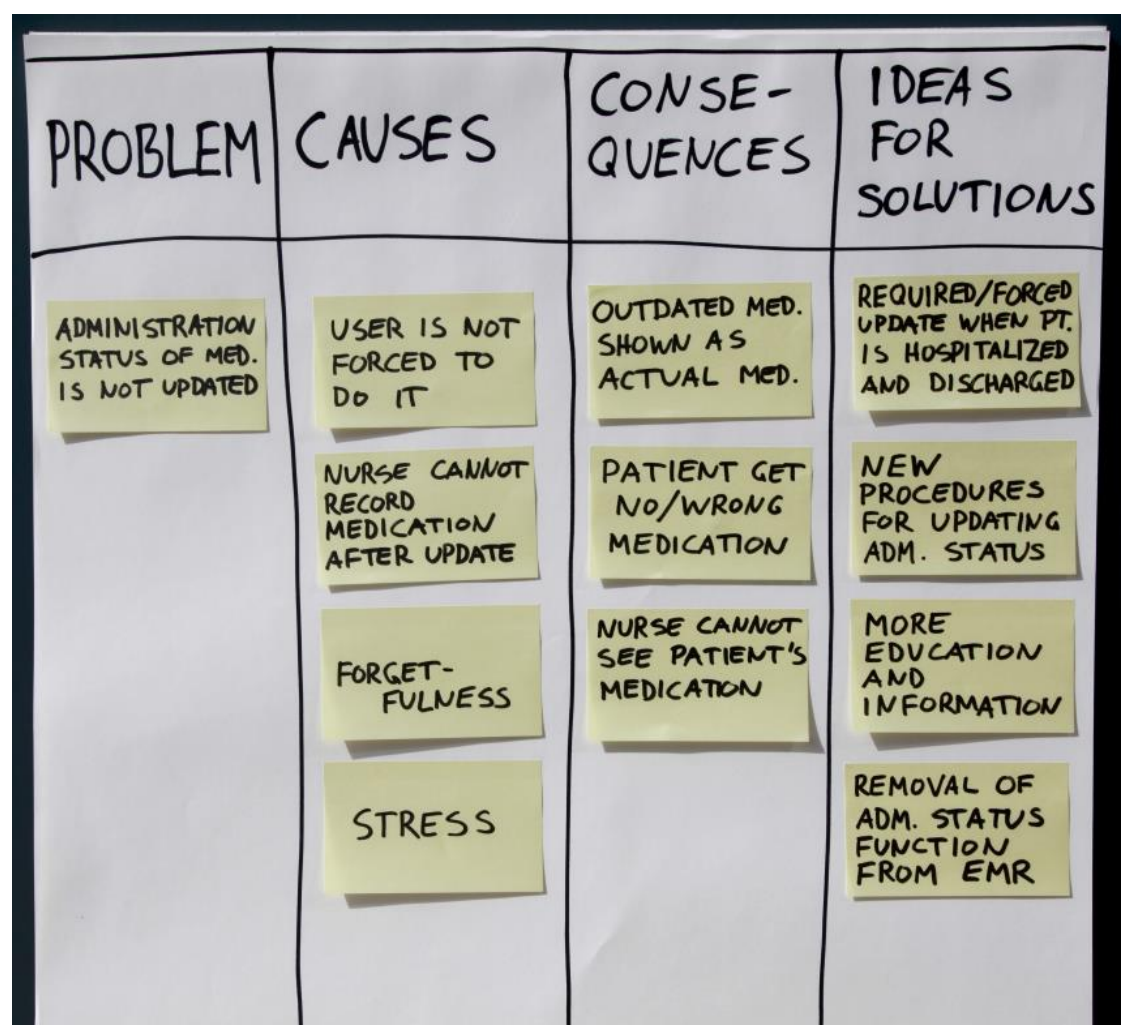

Figure 2: Photo depicting a diagnostic map. The diagnosed problem is further described in the case below.

Common to both affinity diagramming and diagnostic mapping is the use of adhesive notes to visualize codes, categories, and argument chains. These visualizations are the central tools during the workshops where the involved participants meet and utilize the techniques. The visualizations work as an agenda-setting tool through which the dialogue between the participants is mediated: The adhesive notes are continuously made, reviewed, changed, moved, and pointed, and referred to during the discussions and reflections at the workshop. The techniques and the workshop form (as exemplified and described in the following sections) a 'space' for the voice of all participants. The grouping of codes into categories in the affinity diagram, and the structuring of explanations and argument chains in the diagnostic map, are made as a collective effort. Thus, both techniques are designed to support collective analysis where multiple participants share interpretations and mediate their discussions using the visualization tools depicted in Figures 1 and 2 . 


\section{Collective Analysis of a New EMR System}

The case study involved the implementation and use of a new electronic medical record (EMR) system in Region Zealand, one of five health-care regions in Denmark. The system was implemented in order to obtain a safer medication procedure in all hospitals throughout the region. A number of prior studies had shown that quite often there was a significant difference ("noncompliance') between the medication that a patient had been prescribed by the physician and the kind and amount of medicine that the patient actually had taken, and this could counteract with the treatment and harm the safety of the patient (see for example Bates et al. 1995). The reason for this is partly due to multi-pronged documentation of drug prescriptions in the paper-based record and partly due to absent or inconsistent documentation of the actual drug intake. Multi-pronged documentation means that the same prescription is recorded several times at different places in the record, e.g. documented both in the nursing record and in the physician record specific to a single ward. Examples of errors resulting from the use of the EMR system are described by Hertzum (2010).

Political pressure was applied to increase the documentation and control of the medication process. EMR systems are expected to eliminate (or drastically reduce) medication errors by providing a technological platform that ensures single-stranded documentation and an accurate alignment of the patient's prescriptions and actual intake (Bates et al. 2003).

The Zealand region was the first region to complete a large-scale implementation of EMR. The system was implemented during 2003 and 2004, accompanied by a thorough and systematic introduction and training of the clinical staff (which in 2004 had involved 21 man-years). In April 2004 the system had about 2.500 users and they had passed one million medical transactions from more than 20.000 patients. The hospitals in the region handle about 50.000 hospitalizations and 300.000 outpatient treatments every year.

After the implementation, the people in charge of the project (in the following referred to as 'the project group') undertook a questionnaire survey to learn about the user experience during the implementation period and when using the system on a daily basis. Participation in the survey was anonymous and 377 users responded to the survey, including 115 physicians and 220 nurses representing the main users of the EMR system. The response rate was low, most probably due to the fact that the survey with its 127 fixed-response questions took approximately 45 minutes to complete. Nevertheless, the result of the survey included more than 2.200 optional free-text comments, 700 from physicians, 1.300 from nurses, and 200 from 'others.'

It came as a surprise to the project group that the clinicians did not experience the new medication practice as being unequivocally safe: One-third of the respondents reported to the contrary, that the medication procedure had 
become less safe and it sometimes led to life-threatening situations. The immediate design problem in question was: Why is the new EMR-supported medication process not experienced as being unequivocally safer?

While the survey identified a severe design problem, it gave no immediate answer as to why this problem had emerged. The many optional comments entered by the clinicians indicated a high desire to explain in their own words how they had experienced problems using the system. Some of the reasons for the design problem may have been explained in these comments. The project group did not know how to deal with all these 'unstructured' qualitative statements and they asked two researchers (the authors of this chapter) to analyze the statements and look for possible reasons for the problem.

In addition, it was decided to conduct focus group interviews with clinicians in order to let them reflect upon, discuss, and elaborate on their experiences using the system. Four focus group interviews were conducted with ten chief physicians, nine specialist registrars and residents, and seven nurses respectively, as well as one with a mixed group of seven physicians and nurses. One of the authors of this paper participated in the interviews. She had, prior to the interviews, visited a medical ward to observe how the nurses administrated the medicine and how physicians used the system during their ward round. The focus interviews were audio-recorded and transcribed in "a more formal, written style" (Kvale 1996, 170) focusing on passages related to the safety issue.

\subsection{Using Affinity Diagramming}

All the survey statements were printed with a relatively high font size and cut into individual paper notes each representing one statement. By using a large font size it was easier to allow multiple participants to get an overview of them when arranged on a table or on a wall. A primary sorting of the notes reduced the number to approximately 250 different-type statements as a result of sorting out identical (or almost identical) statements and statements that were clearly not related to the safety issue. The selected survey statements and selected statements from the interview were then subjected to constant comparison using the affinity diagramming technique.

The statements were taken one by one, briefly discussed and interpreted by the researchers and then attached to a wall. To begin with, each statement was compared to all the statements on the wall, to assess if it pointed towards one or another of them, and this way groups of statements quickly began to form. A headline for a group was written on colored adhesive notes (see Figure 3). After about a third of the statements had been placed on the wall the process had changed toward an assessment of which group a new statement belonged to. Sometimes a statement could be related to more than one group and this triggered a discussion of how to interpret groups of statements. The groups in the affinity diagram developed during the process and some groups formed 
groups with subgroups (high-level categories with associated low-level categories).

The affinity diagram identified four problems:

1. Administration status is not updated.

2. Medication status is not updated.

3. Medical procedures are circumvented.

4. Medical documentation is multi-pronged.

It is noteworthy in this case that the affinity diagramming identified four general problematic situations of using the new electronic medical system, and that no new problematic situations arose later on in the case. The first three problems are described and explained below (in the section 'The Results of the Analysis'). The fourth category is not further touched upon in this chapter: This problem addressed the systems failure to obtain a single-stranded documentation: that is, that a given medication is recorded once only in the system.

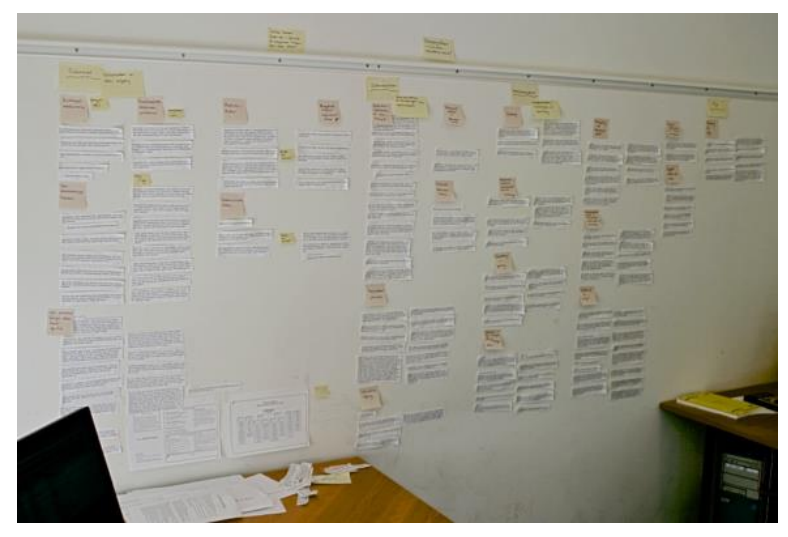

Figure 3: Photo from the process of making the affinity diagram.

\subsection{Using Diagnostic Mapping}

Diagnostic mapping was used to analyze the relationship between the problems of using EMR, their causes, the negative consequences, as well as possible solutions. The starting point for the mapping session was the problems identified in the affinity diagram.

The researchers made a first version of the diagnostic maps and then used these maps during a workshop with the projects group where they were presented, discussed, revised, and elaborated. Problem situations were mapped through diagnostic maps related to the use of the system's administration status, medicine status, and circumvented procedures. The resulting diagnostic maps reflected the researchers' and project group participants' collective analysis and interpretation of the problematic situations, and they were 
subsequently used by the project group to initiate actions to remedy the problems.

The maps were made with adhesive notes attached to flip-over papers that could easily be moved around and revised (see Figure 2 representing a map diagnosing the problem 'Administration status is not updated'). Affinity diagram notes formed part of the input to the map representing this problem. However, there is not a one-to-one relation between notes on the diagnostic map and the affinity diagram. Some notes from the affinity diagram may fit into the map, while the participants, based on their interpretation of the situation, construct others. This was typically the case for notes relating to 'causes' and 'ideas for solutions.'

The ideas for solutions in the maps comprised changes to the technology and clinical procedures as well as education and information related to the EMR system. Figure 4 shows one immediate action taken after the workshop: Informing the clinicians at the emergency departments by drawing attention to the possible risk induced by the EMR system. Readers interested in the efforts and experiences in trying to remedy the problems are referred to Granlien et al. (2008) and Granlien and Hertzum (2012).

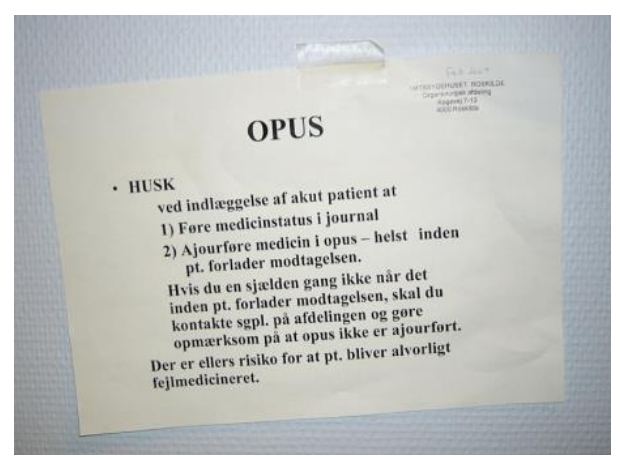

Figure 4: Photo of the 'attention sign' at an emergency department warning the clinicians about a potentially dangerous situation with the EMR system (text in Danish). The sign was made immediately after the diagnostic mapping workshop and reads as follows: "REMEMBER when hospitalizing an acute patient to 1) set the medicine status in the patient record; 2) update the medicine in the system - preferably before the patient leaves the emergency department [and is transferred to another ward]. If occasionally you are not able to make it before the patient is transferred you must contact the nurse at the receiving ward and call attention to the fact that the system is not updated. Otherwise there is a risk that the patient will have seriously inappropriate medication."

\subsection{The Results of the Analysis}

Why did the clinicians not experience the new EMR-supported medication process as being unequivocally safer? While the clinicians do agree that some medication errors and inexpediencies are solved by the system, they also point 
out that new potential dangerous situations emerge. Common to these situations is incorrect use of the system (i.e. incorrect according to the formal medication procedure), which results in erroneous data in the system, which again leads to dangerous situations that are difficult to detect, predict, and prevent.

The new EMR system led to critical medication situations deeply embedded within the complex organization and practice of using the technology. The system entailed a more detailed and less flexible documentation practice. The system enforced workflows that previously were also mandatory but easy to circumvent. New laborious procedures, introduced by the system, were sometimes forgotten, omitted, or circumvented in order to 'get the job done' in a busy situation, while other stricter and obligated procedures were either deliberately opposed or omitted due to confusion about the division of responsibilities among the clinicians.

Below we describe the three aforementioned problematic situations concerning the use of the system's 'administration status,' the 'medicine status' and the related circumvented medical procedures.

The first situation concerns the introduction of a new procedure in terms of updating the drug 'administration status' (indicating who is administrating the patient's drug). This procedure is aimed at reducing medication errors, but the procedure introduces new error situations if not used consistently by all clinicians. The administration status provides the clinician with a functionality to explicitly make up one's mind about who should administer the patient's different types of medicine during and after being hospitalized. The medicine should, among other things, be given the status of either 'none,' 'partial,' or 'full' self-administration, referring to how the patient is in control of taking the medicine. This did not always work as intended, as described by a physician in the story below:

...We discharged the patient from our ward to the outpatient clinic. Treating a patient on an outpatient basis means that the patient, in principle, is in charge of the medicine and the administration status in the system must be set to 'self-administration.' The patient then gets worse and is admitted once again to our ward as an emergency patient. The physician receiving the patient looks into the medical system and notices all the ordinated medicine - that's OK, that's what the patient should have. But he overlooks a little tiny box with a checkmark, which means that the status of the administration is set to 'full self-administration' [see Figure 5]. So the patient is hospitalized but without any medicine prescribed [by the hospital]. The nurse checks whether the patient should have any medicine and cannot see any prescriptions [that she is in charge of - because the patient himself is responsible for taking this]. The error is realized three days later when the patient suffers from an accumulated and severe epileptic seizure. You might claim that this is a human error - that it is not an error in the system - but it really imposes a factor of uncertainty. (citation from focus group interview) 


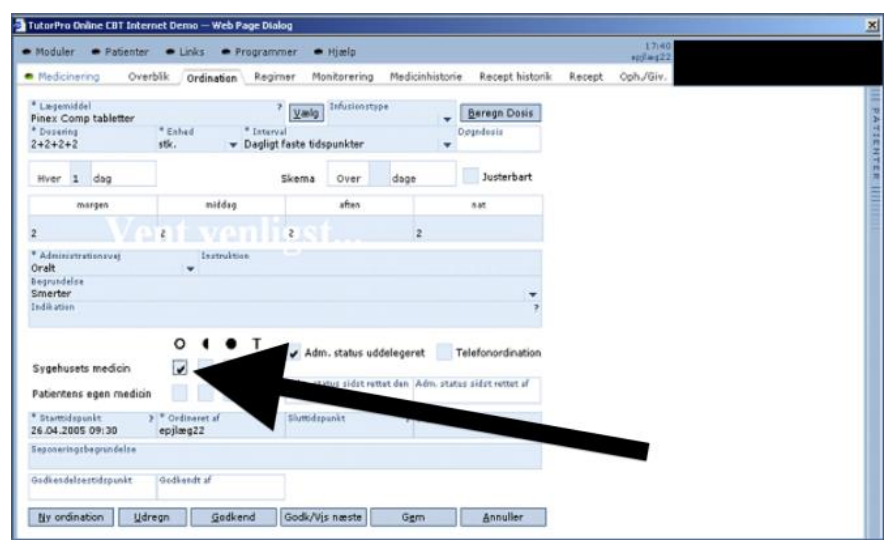

Figure 5: Screen dump from the system with arrow pointing to checkbox with administration status.

The second situation describes how some physicians resist aligning to an enforced procedure related to the 'medicine status.' It concerns a well-known clinical procedure as described by the Danish National Board of Health: That the physician in charge of a patient must be responsible for the complete drug portfolio of that patient. This procedure aims at, among other things avoiding the risk of so-called drug interactions where different drugs change the impact of one another. While this always has been a general rule of attention for the physician, the implementation of the system required the physician to set the 'medicine status,' hereby explicitly recording who and when made this judgment for a given patient:

...I never touch the medicine status - because I work in a psychiatry ward and then [if I touch the medicine status] I become responsible for all the somatic medicine ordinated too. And I don't know anything about that... (citation from focus group interview)

If the physician does not set the 'medicine status' the EMR has failed to resolve a problem that was equally present before its introduction, but in addition new problems might occur as illustrated in the third situation below.

The third situation illustrates that the enforced workflow implies that a correct marking of the administrative and medical status is sometimes forgotten. Before a patient is discharged from the hospital the physician should assess the medicine status and decide which medication is no longer relevant (and mark this as 'discontinued') and which medication the patient should continue to take (and mark this as 'full self-administration'). However, when the physician marks a drug as 'full self-administration' the nurse is no longer allowed to mark that the medication has been given. And sometimes the nurse gives the patient medication just before the patient is discharged. This enforces a procedure where the physician must await any remaining medication before setting the status - and thus this was often forgotten, as illustrated in the following story:

...what they do is actually right [awaiting setting the status until after the patient is discharged] because otherwise the nurse is prevented from doing anything when 
discharging the patient. So they postpone it and this initiates the problem at a subsequent hospitalization. They skip it because it bothers us right now and then we forget to set it [afterwards]... (citation from diagnostic mapping workshop)

A patient was hospitalized at the anesthesia ward and was then discharged [...] without any change recorded on the medicine status. Then [several months later] the patient was hospitalized in our ward and I noticed that the patient was ordinated ten different intravenous drugs. I discontinued all of it... (citation from focus group interview)

The problems described above arose due to the new reconfigurations of existing procedures, organization, and clinical work practices that took place. These reconfigurations aim at making the clinicians' comply with new technologies in terms of the EMR system, as well as the new medication procedures imposed and facilitated by the system. Such intrinsic and complex relationships between information technologies and work practices are far from an exception and resemble other empirical investigations within the health-care domain (for example Berg 1999; Berg et al. 2003).

\section{Hands-On Guidelines to Collective Analysis}

How can a university student group approach a collective analysis of their qualitative data? In this section we present some concrete hands-on guidelines to begin such an analysis. An illustrative overview is given in Figure 6.

A starting point for using the affinity diagramming technique could be that the student group had conducted a number of interviews. Let us, for the sake of this example, picture a situation where you participate in a group with three other fellow students. Your group has conducted five interviews where two or three students together met the interviewees. Each interview was audiorecorded. After each interview one of the students, who participated in the interview, listened through the audio recording and made a transcription (Kvale 1996, 170) (for example, five to ten pages for an interview). The resulting five transcripts were distributed to all of you and you now make your individual preparation for a joint affinity diagramming workshop.

Your preparation comprises your individual interpretation of the interviews. You start by making a printout of all five interview transcripts and sequentially mark them with a number and your initials. You now read them, and mark all words, phrases or short passages where you observe something of special interest to you (for example using a highlighter felt-tip pen). Then you go through all the markings and make, for each of them, a relevant headline on an adhesive note with a large pen. The headline on each adhesive note should be readable from a distance when the group meets and looks at the affinity diagram. On each adhesive note you also make a small referential comment in the lower-right corner with your initials, number of the interview, and the page number where the passage that this adhesive note refers to (e.g. "AJ, int. \# 3, 
p. 5"). This way you can look up in the interview transcription printout and see the context for the specific code on each of the adhesive notes.

When your group meet for the affinity diagramming workshop all of you bring your annotated interview printouts and your pile of adhesive notes. During the workshop your collective analysis is initiated, based on each of your individual interpretations. If it's your first time making an affinity diagram you might experience the significant, and positive, difference from how you have worked with qualitative material beforehand, or, as the inventor of affinity diagramming might phrase it: "Breakthrough in traditional concepts is needed. When the only solutions are old solutions, try an Affinity to expand the team's thinking" (Brassard 1989, 18).

You go through the interviews one by one. One of you who conducted the interview starts and goes through all your observations one by one: You take each adhesive note, put it on the whiteboard or wall in front of you and explain briefly what this code is about and why you chose it. The others might ask clarifying questions. Hereafter, in turn, the other group participants go through their observations and put up their adhesive notes on the wall placed near to similar notes and categories that emerge during the process. When making the affinity diagram, headlines are considered at the same time for the emerging categories, noted on new adhesive notes and placed above the group of notes they belong to.

When all of you have gone through all your notes you have an affinity diagram structured in categories. You may also have a portion of notes that do not belong to any specific category: They can be grouped together in one group headlined 'the fridge.' The fridge can help you concentrate on the notes you find interesting while not having a feeling of ignoring other notes since they are 'preserved' in the fridge and you can return to these later, if needed. The categories might form the sections of a subsequent report. Writing this report may be distributed among you so each of you makes a draft of some of the categories. The notes for each category constitute the headwords to consider. If you question the meaning or context of a headword, you can use its reference to return to the interview summary to which it belongs.

Diagnostic mapping is a relevant technique after one or more affinity diagrams have been made. You can use the technique to establish an overview and joint understanding of a problematic situation, while also noting possible courses of action for solving the situation. It allows your group and others that you collaborate with to express and solidify their statements, interpretations, and ideas for solutions regarding a situation that you want to improve. Diagnostic mapping can help you to visualize your argument chains that explain how you interpret a problem and how it might be solved. The map supports you in explicating your abductive hypotheses and candidates (guesses) of explanations so they can be collectively reviewed. Two typical situations using diagnostic mapping could be: 
- As a follow-up to the affinity diagramming of your interviews. The affinity diagram might reveal a number of problematic situations you would like to investigate further. This may involve mapping such situations in collaboration with the interviewees or others that you collaborate with.

- As a tool for your project group for outlining and establishing an overview of the total volume of problems, needs, and solution proposals that your project has revealed so far - in order, for instance, to assist you in a prioritization. (Bødker et al. 2004, 278f)

As with affinity diagramming the mapping is done in a joint workshop where you engage in a process of collectively analyzing the problematic situations. In preparing the workshop you should agree on what problematic situations you want to analyze. And, just as with affinity diagramming, you should make an individual interpretation of these problematic situations by outlining your own diagnostic maps prior to having the workshop. The individual preparation is important for both techniques: If you skip the individual work this might outweigh what is gained by working collectively.

The mapping is done on a wall or on big sheets of paper on the wall with four (empty) columns headed: Problem, Causes, Consequences, and Ideas for Solutions. Using sheets of paper from a flip-over helps you make a map that you can easily move and take with you. Each problematic situation that is being studied provides the title of one map.

Start by listing the possible interpretations of the problematic situation, that is, problem candidates in the left-hand column. Write down all the suggestions in the problems column as short statements that fit into the adhesive notes. Let the group member who suggested the problem propose the wording. You may help define the problem, but it is not very helpful if you merely reinterpret or rephrase the problem. Use adhesive notes just like in affinity diagramming so it is easy to move a suggestion to a different spot on the map. Often, something that initially is perceived as a problem later turns out more preferable to constitute a cause or a consequence.

Next, take up the problems one by one and outline the causes, consequences, and potential solutions for each. It might help to ask questions like:

- What is causing the problem? What are the conditions and reasons behind having this problem?

- What are the most negative consequences of the problem - those we are unwilling to accept? What consequences and additional problems does the original problem entail?

- What potential solutions to the problem can we come up with? What do we imagine would eliminate or remedy the problem? 
Codes from the affinity diagrams might form some of the candidates for input to the diagnostic map but more often the affinity diagram forms a basis for making the diagnostic map rather than any kind of one-to-one transfer of adhesive notes from the diagram to the map. In our experience, the qualitative data from interviews etc. often form observable input to the problems and consequences columns, while the participants making the map interpret and suggest most of the input to the causes and ideas for the solutions column. Reviews of diagnostic maps made together with a partner of yours thus often challenge the hypotheses of causes and provide new innovative candidates for interventions (for an example of this see Simonsen 2007).

If the consequences of a problem are few, or there are no suggested unacceptable consequences, the problem may be superficial - that is, the interpretation of the problematic situation is irrelevant. If, on closer consideration, the consequences of a problem are found to be bearable, the problem probably does not warrant further study.

It may be hard to distinguish among problems, consequences, and causes. The consequences of a problem are perhaps seen as constituting problems in themselves. If a consequence of a problem is regarded as a significant separate problem, we recommend putting it down on the list as a new problem.

Diagnostic mapping is an effective technique for the project group to discuss and agree on how to interpret and intervene in problematic situations. It is equally effective to bring your analysis to your collaborating partners, just as the researchers brought their diagnostic maps to the project group in the case of the EMR system described earlier in this chapter. We generally advise that you make a diagnostic map on your own that you present and revise together with your partner (see Simonsen (2007) for an elaborated example for how to do this). If you have a very trustful relationship with your partner(s) you might also consider making the diagnostic map together, from scratch.

\section{Conclusion}

This chapter has presented a method as a resource for situated action, specifically oriented towards facing large amounts of qualitative data: The objective is to traverse the empirical data and distill the relevant issues and problems as part of a design process. The two presented techniques cannot direct any exact pathway to follow but they can form a concrete resource for the project group to embark on a collective analysis of the qualitative data.

In the case presented, the qualitative data came from two sources: (1) free-text comments from a survey originally designed to make an overall evaluation of the EMR system, its use, and the implementation process, and (2) from transcriptions from focus group interviews where clinicians' reflected on their experiences using this system. The techniques might be used with qualitative data from other sources too: for example, from observations or from 
collections of documents. We explained how the data initially were analyzed through an inductive and Grounded Theory-inspired approach using affinity diagramming as a simple yet effective technique to manage large qualitative data material. The result of the affinity diagramming process identified, characterized, and generalized the problems. The affinity diagramming was followed up by the diagnostic mapping technique. This technique undertakes an abductive approach by forming hypothetical explanations to the problems from the affinity diagram as well as suggesting possible interventions that might solve the problems. Both techniques are based on making the data and the participating actors' interpretations of the data highly visible using adhesive notes. During the workshops, this visualization mediates and supports the collective discussions and reflections taking place among the participants.

Thus, the chapter demonstrates how to combine techniques to manage a process of descriptive analysis and proactive intervention activities. An overview of the problem was done through affinity diagrams while diagnostic mapping was used to facilitate workshops aimed at problem solving and devising ideas for interventions. This collective analysis is summarized in Figure 6. 


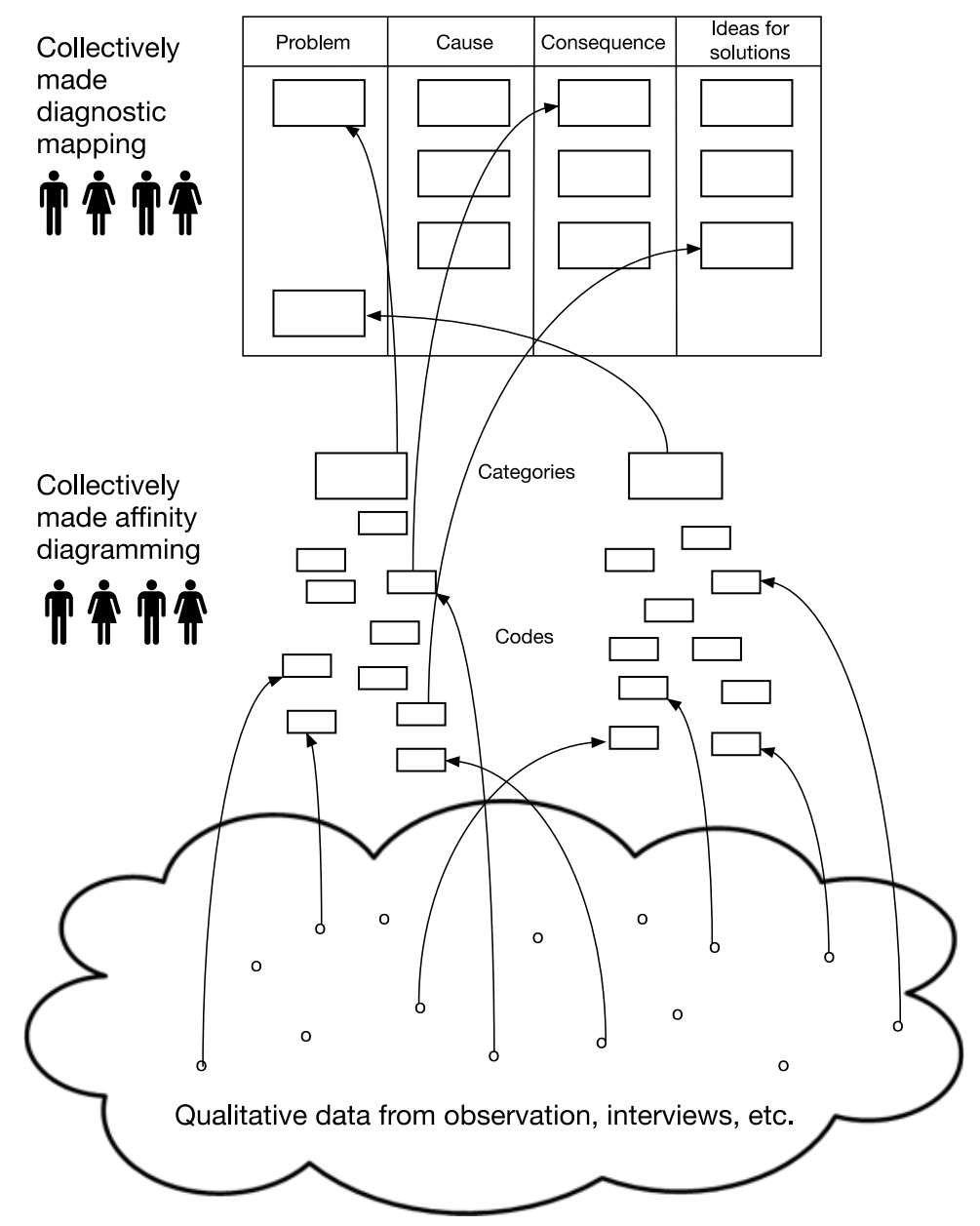

Figure 6: Collective analysis of qualitative data by combining affinity diagramming and diagnostic mapping. Codes are made from the qualitative data forming input to the affinity diagram where the codes are grouped in categories. Categories identifying problems form initial input to diagnostic maps where problems are explained, and solutions suggested. The map is constructed by the participants, based on their interpretation and hypotheses. Codes might form part of the input to the diagnostic maps.

\section{References}

Andersen, Niels Erik, Finn Kensing, Jette Lundin, Lars Mathiassen, Andreas Munk-Madsen, Monika Rasbech, and Pål Sørgaard. Professional systems development: Experience, ideas and action. New York: Prentice Hall, 1990.

David W. Bates, David J. Cullen, Nan Laird, Laura A. Petersen, Stephen D. Small, Deborah Servi, Glenn Laffel, Bobbie J. Sweitzer, Brian F. Shea, Robert Hallisey, Martha Vander Vliet, Roberta Nemeskal, Lucian L. Leape, David Bates, Patricia Hojnowski-Diaz, Stephen Petrycki, Michael 
Cotugno, Heather Patterson, Mairead Hickey, Sharon Kleefield, Jeffrey Cooper, Ellen Kinneally, Harold J. Demonaco, Margaret Dempsey Clapp, Theresa Gallivan,, Jeanette Ives, Kathy Porter, B. Taylor Thompson, J. Richard Hackman, and Amy Edmondson. Incidence of adverse drug events and potential adverse drug events: Implications for prevention. JAMA 274, no. 1 (1995): 29-34.

Bates, D.W., G.J. Kuperman, S. Wang, T. Gandhi, A. Kittler, L. Volk, C. Spurr, R. Khorasani, M. Tanasijevic, and B. Middleton. Ten commandments for effective clinical decision support: Making the practice of evidence-based medicine a reality. Journal of the American Medical Informatics Association 10, no. 6 (2003): 523-30.

Berg, M. Accumulating and coordinating: Occasions for information technologies in medical work. Computer Supported Cooperative Work 8, no. 4 (1999): 373-401.

Berg, M., J. Aarts, and J. van der Lei. ICT in health care: Sociotechnical approaches. Methods of Information in Medicine 42, no. 4 (2003): 297301.

Beyer, Hugh, and Karen Holtzblatt. Contextual design. Defining customercentered systems. San Francisco, CA: Morgan Kaufmann Publishers, Inc., 1998.

Brassard, Michael. The memory jogger plus. Featuring the seven management and planning tools. Methuen, MA: GOAL/QPC, 1989.

Bødker, Keld, Finn Kensing, and Jesper Simonsen. Participatory IT design. Designing for business and workplace realities. Cambridge, MA: MIT Press, 2004.

Glaser, B. Basics of grounded theory analysis: Emergence vs. forcing. Mill Valley, CA: Sociology Press, 1992.

Glaser, Barney G., and Anselm L. Strauss. The discovery of grounded theory: Strategies for qualitative research. New York: Aldine de Gruyter, 1967.

Granlien, M. F., M. Hertzum, and J. Gudmundsen. The gap between actual and mandated use of an electronic medication record three years after deployment. In eds. S.K. Andersen, G. O. Klein, S. Schulz, J. Arts and M. C. Mazzoleni, MIE2008: Proceedings of the XXIst International Congress of the European Federation for Medical Informatics, Göteborg, Sweden May 25-28 (2008): 419-424.

Granlien, Maren Sander, and Hertzum, Morten. Barriers to the adoption and use of an electronic medication record. Electronic Journal of Information Systems Evaluation 15, no. 2 (2012): 216-27.

Hertzum, Morten. Breakdowns in collaborative information seeking: A study of the medication process. Information Processing \& Management 46, no. 6 (2010): 646-55.

Kvale, Steinar. Interviews. An introduction to qualitative research writing. Thousand Oaks, CA: Sage Publications, 1996.

Lanzara, Giovan Francesco, and Lars Mathiassen. Mapping situations within a system development project. Information and Management 8, no. 1 
(1985): 3-20.

Simonsen, Jesper. Involving top management in IT projects: Aligning business needs and IT solutions with the problem mapping technique.

Communications of the ACM 50, no. 8 (2007): 53-58.

Suchman, Lucy A. Human-machine reconfigurations: Plans and situated action. 2nd ed. Cambridge, New York: Cambridge University Press, 2007. 